\title{
Un processus postcolonial :
La genèse de la domination kényane
dans les courses de fond vue par la presse
sportive spécialisée française
}

Cyril Thomas

Docteur en STAPS

Université Lyon 1

$L-V I S$

France

cyril.thomas42@hotmail.fr

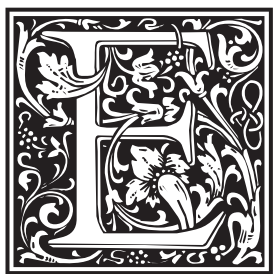

xceptionnellement prolifique en matière de courses sur route, la période s'étendant de septembre 2019 à janvier 2020 voit le premier homme courir un marathon en moins de deux heures, dans une épreuve non officielle ${ }^{1}$, et cinq records du monde améliorés sur des distances allant du $5 \mathrm{~km}$ au marathon. Cette succession d'exploits, tous réalisés par des athlètes est-africain-es, pour la plupart kényan $\cdot e^{2}$, suscite bien entendu l'intérêt d'une presse sportive désormais largement coutumière de la domination des athlètes originaires de ce pays dans les courses de fond ${ }^{3}$. Si les médias alimentent à ces occasions le débat sur la légitimité des différents avantages $^{4}$ dont bénéficient les athlètes pour réaliser ces performances indépendamment de leur origine géographique, tel n'a pas toujours été le cas. L'avènement des athlètes kényan.es dans les courses d'endurance, depuis 1960, est en effet largement questionné par la presse sportive, désireuse de comprendre et d'expliquer le «phénomène kényan $»^{5}$.

L'objet de cet article est de montrer que l'éclosion des athlètes kényan-es au plus haut niveau international dans la période post-coloniale est appréhendée par les journalistes français selon une rhétorique s'inscrivant dans un processus postcolonial. Si l'adjectif post-colonial paré d'un trait d'union incarne une dimension uniquement chronologique, renvoyant à la période qui suit les indépendances, l'absence de trait

Pour citer cet article, to quote this article, para citar este artigo :

Cyril Thomas, «Un processus postcolonial : La genèse de la domination kényane dans les courses de fond vue par la presse sportive spécialisée française. », Sur le journalisme, About journalism, Sobre jornalismo [En ligne, online], Vol 10, n² - 2021, 15 décembre - december 15 - 15 de dezembro. URL : https://doi.org/10.25200/SLJ.v10.n2.2021.447 
d'union renvoie aux postcolonial studies, un courant de recherche qui s'intéresse aux effets de la colonisation sur les sociétés actuelles, transcendant les périodes pré-coloniale, coloniale et post-coloniale (Savarese, $2014: 32$ ). La notion de processus postcolonial intègre les transformations qu'ont subies des phénomènes ancrés dans la période coloniale, ayant des répercussions dans la période actuelle, sur les sociétés des ex-métropoles comme celle des anciennes colonies (Blanchard et Bancel, 2006 : 14). Si le Kenya, ancienne colonie britannique, n'a jamais appartenu à l'empire colonial français, les modalités discursives selon lesquelles les journalistes sportifs français couvrent les succès de ses athlètes dès les années 1960 sont le reflet de la domination culturelle caractérisant la relation coloniale (Forté, 2019 : 77). Parce que les situations postcoloniales ne sont pas une simple reproduction des faits coloniaux, ce rapport de domination gagne en réciprocité à mesure que les athlètes kényan·es s'imposent au sein de l'athlétisme mondial. Ces dernier-es opposent une domination sportive à l'hégémonie culturelle européenne héritée de la colonisation, obligeant à s'extraire d'un simple rapport binaire colon/colonisé (Smouts, 2007 : 43). Point de départ de cette étude, l'année 1960 marque l'entrée de l'athlétisme est-africain dans le concert international, l'Ethiopien Abebe Bikila triomphant cette année-là aux Jeux olympiques de Rome d'un marathon chargé de symboles ${ }^{6}$ devant les caméras de télévision du monde entier (Gaudin, $2008: 74$ ).

Pour mener cette étude, nous avons investigué trois revues françaises spécialisées dans l'athlétisme, paraissant dans les années 1960, au moment où le Kenya se révèle au plus haut niveau international : L'Athlétisme, Le Miroir de l'athlétisme et l'Équipe athlétisme magazine ${ }^{7}$. Rompant avec le traitement factuel de l'information en vigueur dans la presse sportive quotidienne, la mise en récit propre au format magazine permet de saisir les représentations des acteurs ainsi que leurs transformations (Attali, 2018 : 179-18o). Le choix des revues s'est porté d'une part sur leur réputation de références concernant l'athlétisme dans les années $1960^{8}$, ainsi que sur l'hétérogénéité de leurs textes (Krieg 2000) en lien avec l'éclectisme de leurs lignes éditoriales. Créée en 1921, la revue l'Athlétisme, devenue Athlétisme à partir de 1978, est ainsi l'organe de presse officiel de la Fédération Française d'Athlétisme (FFA). Essentiellement tournée vers l'athlétisme français, on y trouve des articles, des résultats ainsi que des comptes rendus des instances dirigeantes de la FFA. Rebaptisée Athlétisme Magazine depuis 2001, c'est la seule parmi les revues étudiées à paraître encore actuellement. La seconde revue, Le Miroir de l'athlétisme, publiée de 1962 à 1974 sous la direction de Yann le Floch, est un mensuel spécialisé décliné du journal Miroir Sprint dirigé par Maurice Vidal de 1946 à 1971. Défini comme « un hebdomadaire sportif d'obédience communiste » (Bazoge et Jamain-Samson, $2013: 233$ ), ce journal édite dans les années 1960 plusieurs autres revues spécialisées dans différents sports. Éditée de 1968 à 1975 par le journal l'Équipe, alors dirigé par Gaston Meyer, L'Équipe athlétisme magazine, comme le Miroir de l'athlétisme, est une revue spécialisée davantage tournée vers l'athlétisme international. La proximité avec la revue précédente s'arrête néanmoins là, puisque l'Équipe et Miroir Sprint sont plutôt opposés idéologiquement, le premier nommé défendant son apolitisme devant les critiques de son concurrent (Seidler, 1964 : 179). L'implication de Gaston Meyer dans la revue fédérale dans les années 1930 ainsi que la participation de certains journalistes à la rédaction de l'Équipe Athlétisme Magazine et de l'Athlétisme témoignent de leur proximité ${ }^{9}$. La revue l'Athlétisme consacre une place importante aux dirigeants fédéraux dans ses colonnes, tandis que le Miroir de l'athlétisme fait intervenir régulièrement des entraineurs, athlètes ou observateurs de l'athlétisme étrangers ou des journalistes de Miroir Sprint dont l'athlétisme n'est pas la spécialité. Cette variété de rédacteurs est autant représentative du « rapport intime avec l'objet traité » qu'entretiennent les journalistes sportifs, que de leur capacité à « couvrir indifféremment différents sports » (Souanef, 2019 : 191-192). Une lecture analogique des articles et des rubriques traitant de l'athlétisme kényan a pu être exercée après avoir retenu de manière synoptique tous les textes concernant l'athlétisme africain. L'analyse porte sur le discours dans son ensemble, intégrant le contenu et les stratégies énonciatives, permettant d'en saisir la fonction culturelle reliant les revues à leur lectorat (Provenzano, 2018 : 89-90).

Le corpus établi nous a permis de saisir les différentes évolutions dans l'appréhension par la presse spécialisée de la domination kényane dans les courses de fond à travers quatre étapes chronologiques. Afin de ne pas nous limiter à une histoire descriptive (Farge, 1989: 91), nous avons interrogé ce corpus sous l'angle de la notion de domination qui caractérise la période coloniale, entre sujétion et mission civilisatrice, se traduisant par « un double discours [...] à la fois intégrateur et discriminatoire » (L'Heuillet, 2017 : 42). Ainsi, nous avons observé que bien que le Kenya n’ait jamais été colonisé par la France, les journalistes français appréhendent la montée en puissance des athlètes kényan-es selon les mêmes modèles rhétoriques que leurs homologues britanniques à la fin de la période coloniale : la surveillance (comparaison des athlètes kényan·es aux athlètes européen·nes), l'appropriation (perception des athlètes selon le regard européen), la négation (mobilisation de prétextes comme l'altitude pour minimiser les performances des athlètes kényan·es) et l'idéalisation (image de l'athlète pourvu.e d'un avantage naturel perçu comme injuste) (Bale, 2001 : 224-228). Tandis que les postcolo- 
nial studies s'intéressent essentiellement aux relations entretenues entre les anciennes métropoles et leurs ex-colonies (Lacoste, $2006: 15$ ), nous prenons donc le parti d'élargir la question postcoloniale aux interactions culturelles germant entre deux pays dépourvus de liens coloniaux.

\section{0-1968 : SPÉCULATION SUR UN POTENTIEL ATHLÉTIQUE}

Si la victoire d'Abebe Bikila sur le marathon des Jeux de Rome en 1960 est couverte par les médias internationaux, dont les journalistes français, il semble qu'elle ouvre simplement une porte, sans toutefois susciter un véritable engouement médiatique.

C'est après les Jeux olympiques de Tokyo en 1964 que les journalistes français commencent réellement à s'intéresser aux performances des athlètes d'Afrique de l'Est, et plus particulièrement des Kényans. En 1965, le Miroir de l'athlétisme publie ainsi un article proposant un état des lieux de l'athlétisme au Kenya, suite à la première médaille olympique kényane décrochée par Wislon Kiprugut sur 80om à Tokyo. L'auteur prédit alors que « l'athlétisme kényen qui n'en est qu'à ses balbutiements (jusqu'en 1964 l'entrainement se faisait au gré de chacun) a d'énormes possibilités $\gg^{10}$. Deux ans plus tard, après que Kipchoge Keino ait battu les records du monde du 50oom et du 3000 en 1965 , la même revue acquiert les droits de reproduction d'un article paru dans la revue spécialisée britannique World sports. Rédigé par l'entraineur britannique John Velzian, cet article rapporte l'histoire du développement de l'athlétisme au Kenya et identifie des facteurs explicatifs des performances des coureurs kényans. L'auteur évoque alors des facteurs physiologiques, environnementaux et socio-culturels, propres notamment à l'ethnie des Kalenjins ${ }^{11}$, au sein de laquelle émerge la plupart des champions du pays (Manners, 1997 : 14). L'exhaustivité de cette approche multifactorielle, originale à cette époque, tient notamment à l'implication de John Velzian dans l'athlétisme kényan depuis la fin des années 1950, comme instructeur d'éducation physique à l'école de formation des enseignants, puis de la police, avant de devenir entraîneur de l'équipe nationale (Bale et Sang, 1996 : 112). La diffusion de ce sujet témoigne d'une volonté, dès la fin des années 1960, de comprendre les récents succès des coureurs kényans, qui interrogent avant les Jeux olympiques de Mexico. Moins tournée vers l'athlétisme international, la revue fédérale ne produit pas d'articles d'investigation de cet ordre à l'époque, se contentant de faire apparaître au sein des procès-verbaux de comités directeurs une volonté de la FFA d'entrer en relation avec la fédération kényane (Thomas et al., 2021: 93).
Au cours de la période 1960-1968, l'athlétisme kényan commence donc à susciter l'intérêt de la presse spécialisée selon laquelle un certain potentiel se dessine, préfigurant une relation postcoloniale beaucoup plus réciproque que la relation coloniale, culturellement à sens unique (Lacoste, 2006 : 15). Les Jeux olympiques de Mexico en 1968 marquent un premier tournant dans cette perception.

\section{8-1972 : LA DOMINATION KÉNYANE EN QUESTION}

« Les coureurs kényans - qui gagnèrent plus de médailles que la représentation masculine soviétique pourtant réputée la seconde du monde en valeur absolue - achevèrent une phase de l'indépendance kényane à Mexico $»^{12}$.

La verve de Jo Viellvoye, journaliste sportif allemand ayant écrit pour le Miroir de l'Athlétisme, est autant représentative du caractère hyper-appréciatif du discours du journalisme sportif (Vanoudheusden, 2013) que de l'engouement des médias français et étrangers devant les performances des coureurs kényans aux Jeux olympiques de 1968. Seulement composée de quinze athlètes, la délégation kényane y brille, remportant huit médailles, dont trois d'or, sur des distances allant du relai $4 \mathrm{x} 400 \mathrm{~m}$ au $10000 \mathrm{~m}$. Tout comme la victoire de Bikila en 1960 pour l'Éthiopie, la médiatisation de ces performances donne au Kenya une forte visibilité internationale, seulement cinq ans après le retrait des colons britanniques du pays. Les revues spécialisées françaises s'intéressent alors de près aux glorieux champions de la campagne mexicaine, au premier rang desquels Kipchoge Keino, champion olympique du $1500 \mathrm{~m}$ et second du 5000m, devenu un modèle national dans le pays est-africain. Dans un contexte politique fort où les champions acquièrent une dimension universelle (Duret, 1993 : 41-45), la presse internationale lui confère un statut héroïque selon un imaginaire occidental (Bale 2001 : 222-223). Noël Couedel, journaliste à l'Équipe, rédige ainsi un article dans lequel il raconte sa rencontre avec le champion kényan ${ }^{13}$, abordant son enfance, sa carrière et ses projets tout en distillant des éléments emprunts d'exotisme ${ }^{14}$. Ce type de discours, qu'Edward Saïd qualifie d'orientaliste caractérise la façon dont les nations européennes ont créé l'Orient, forme d'exotisme désignant le reste du monde sur laquelle elles portent un regard dominant et s'imposent comme une autorité légitime (Saïd et al., 2005:17-18).

Si le potentiel des athlètes africains éclate au grand jour à Mexico, il ne semble toutefois pas convaincre pleinement tous les observateurs. Certains voient, à travers cette déferlante de médailles, le rôle avanta- 
geux joué par l'altitude à laquelle se situe la capitale mexicaine, proche de celle des hauts plateaux kényans. Si certains médias internationaux vont jusqu'à parler d'injustice où d'humiliation pour les amoureux de courses d'endurance (Bale, 2007 : 22), l'Équipe athlétisme magazine et le Miroir de l'athlétisme se contentent d'installer un certain scepticisme quant à la valeur de ces performances. Noël Couedel relève ainsi que « ce fut évidemment beaucoup trop pour que l'altitude ne fut pas directement responsable de la majorité de ces succès $\gg .{ }^{15}$ Au cœur des débats sur les plans scientifique, médiatique et institutionnel à la fin des années 1960 (Kasperowski, 2009), l'influence de l'altitude est également soulevée à travers la plume de Jean Claude Sorlo, pour lequel « les brillants résultats des pays anglophones en terre mexicaine, favorisées par l'altitude, ne doivent pas faire oublier les performances des nations francophones qui intéressent toute la gamme des spécialités athlétiques ». Le journaliste nuance par l'argument topographique la réussite des pays africains anglophones face aux pays africains francophones, réactivant, via un tableau comparatif, une compétition entre anciens empires coloniaux. Les performances africaines sont à nouveau perçues sous un filtre orientaliste, servant « la force relative des nations concurrentes » (Pociello, 1998 : 164), pérennisant un rapport hégémonique après les indépendances. Cette continuité avec la période coloniale dans l'esprit du journaliste du Miroir de l'athlétisme, s'inscrit dans le virage opéré après les Jeux olympiques de Berlin par la presse communiste, pour laquelle « il n'est plus question de promouvoir l'indépendance des peuples colonisés mais de défendre leur pleine et entière qualité de représentant de la nation sur la scène sportive » (Sabatier, $2013:$ 95).

Ces extraits illustrent le climat de la période de 1968 à 1972. Selon une posture culturellement dominante, les journalistes français se permettent d'interroger la véritable portée des performances des athlètes kényans, pourtant indéniables. A l'origine de ce scepticisme, les théories du déterminisme environnemental, associant les comportements des humains à l'environnement dans lequel ils évoluent, sont soutenues par certain-es scientifiques pendant la période coloniale (Bale, 2002 : 147-158). Cette approche est cependant rapidement tempérée. Moins d'un an avant les Jeux olympiques de Munich, suite aux performances des Kényans aux Jeux du Commonwealth d'Édimbourg en 1970, puis de la réunion pré-olympique l'année suivante, Yves Pinaud, journaliste spécialiste de l'athlétisme africain ${ }^{16}$, prévoit qu' « en athlétisme, l'avenir est à l'Afrique, Munich permettra de le constater et c'est bien là un des intérêts majeurs des $17^{\text {ème }}$ jeux olympiques $»^{17}$. Ce sont également parfois les lecteurs qui s'indignent de la remise en cause de la valeur des athlètes africains par les journalistes, à travers la rubrique qui leur est consacrée dans l'Équipe athlétisme maga- $z_{i n e^{18}}$. Le Miroir de l'Athlétisme publie quelques mois avant cet événement un article rédigé par le Kényan Philippe N'Doo, finaliste olympique sur 10ooom aux Jeux du Commonwealth deux ans plus tôt et journaliste au Daily Nation à Nairobi. A la fois juge et parti, l'auteur débute son plaidoyer au titre univoque en affirmant que « les athlètes africains auront à cœur de démontrer que les médailles qu'ils gagnèrent à Mexico n'étaient pas dues à un "accident géographique" mais bien à leur mérite ${ }^{19}$. La conclusion de l'article, « Nul doute que nous vaincrons ! », illustre finalement la défiance de l'auteur quant au scepticisme des journalistes occidentaux.

Les quatre années qui séparent les Jeux olympiques de Mexico de ceux de Munich révèlent donc autant la mobilisation de théories étroitement liées aux idéologies colonialistes pour expliquer les performances kényanes que la pérennité d'un rapport de domination qui tend à se transformer. Tout en imposant leur appréhension culturelle des premiers succès kényans, les journalistes sportifs français assistent à l'émergence d'une domination sportive, d'autant plus prégnante que s'amorce «la crise du demi-fond français $»^{20}$, qu'ils s'efforcent de contenir par leurs explications environnementalistes. Loin de l'altitude de la capitale mexicaine, les Jeux olympiques de Munich marquent un nouveau tournant dans la perception des athlètes kényans par les journalistes français.

\section{2-1990 : LA CONFIRMATION D'UNE DOMINATION DES ATHLÈTES KÉNYANS}

La période qui suit les Jeux olympiques de Munich laisse apparaître une confirmation du potentiel des coureurs kényans, qui réitèrent leur domination sur les épreuves de demi-fond, sans que l'altitude ne joue directement un rôle, dans différentes compétitions internationales. Devant des performances réalisées au niveau de la mer, les journalistes ne peuvent plus douter de leur suprématie. Pour Mahjoub Faouzi, journaliste et auteur spécialiste du football africain, alors rédacteur pour le Miroir de l'athlétisme :

« Puis vinrent les récoltes de médailles, à Mexico, en 1968, et à Munich, en septembre dernier, avec, s'intercalant entre ces deux événements, les jeux du Commonwealth de Kingston (1966) et d'Édimbourg (1970). L'élite de l'Afrique orientale bouleversa, à chaque manifestation, la hiérarchie et s'intégra dans le peloton de tête. $\gg^{21}$

Nouvellement responsable du service statistique de la FFA, Jean Gilbert évoque quant à lui dans un article pour la revue fédérale « l'arrivée au firmament des coureurs kényans $\gg^{22}$. Le doute ne semble alors plus 
permis quant à la domination des coureurs kényans sur le demi-fond mondial. S'affirmant parmi l'élite internationale, le Kenya devient aux yeux des journalistes un pays qui pèse sur l'échiquier mondial. En témoigne la retranscription des résultats des Jeux olympiques de Montréal puis de Moscou, où plane l'ombre du Kenya bien que le pays ait boycotté ces événements.

Les journalistes sportifs interrogent alors cette hégémonie et tentent de l'expliquer à travers des articles chargés de préjugés racialistes ${ }^{23}$, s'ajoutant aux théories environnementalistes déjà évoquées. Alain Billouin, responsable des rubriques 'Athlétisme' et 'sports olympiques' à l'Équipe, se demande :

« Le Noir a-t-il plus de possibilités que le blanc ? Les conditions géographiques et climatiques sontelles à l'origine de pareilles dispositions ? Les caractéristiques ethniques, en un mot le style de vie africain ont-ils une influence directe favorable ? Oui. Incontestablement. Lorsque la France produit un Jazy et un Wadoux, un pays comme le Kenya peut déposer sur le vaste marché de l'athlétisme une cinquantaine de coureurs de premier plan dont les facilités sont tout simplement renversantes $\gg .{ }^{24}$

Cette période marque, dans les commentaires des journalistes, une rupture dans la perception du coureur africain 'noir' par rapport au coureur européen 'blanc', débouchant sur des théories basées sur des croyances évoquant la génétique et l'idéologie du don. Cette rupture est d'autant plus prégnante que certains journalistes y voient une remise en cause d'un ordre socio-racial jusqu'alors préservé dans l'imaginaire médiatique concernant les épreuves d'endurance (Wiggins, 1989). C'est le cas de l'ancien médaillé olympique Marcel Hansenne, reconverti comme journaliste, d'abord au journal J (devenu Miroir Sprint) puis à l'Équipe, pour lequel « certains observateurs se demandent en effet si ce n'en est pas fini de la supériorité de la race blanche sur les distances allant du $800 \mathrm{~m}$ au $10000 \mathrm{~m} \gg .{ }^{25}$

Fondée sur des critères ségrégationnistes, ce type de rupture n'est pas sans rappeler la reconnaissance par les journalistes de la supériorité athlétique des athlètes noirs, notamment dans les épreuves de vitesse et de détente, qui suit les Jeux olympiques de Berlin près de quarante ans auparavant, suite aux performances des athlètes américains dans le sillage de Jesse Owens. Dès lors, comme elle le fait après les Jeux de Berlin, puis dans une moindre mesure à la fin des années 1950, la presse française recourt à des justifications «pseudo-scientifiques » de ces performances (Deville-Danthu, 1997 : 319). L'idéologie du don naturel est ainsi remise au goût du jour :

« Il a été aussi constaté que les Kalenjins ont un plus long fémur que celui des hommes des autres tribus du
Kenya ! Ils ont donc une plus grande foulée par rapport à leur taille. Les Kalenjins sont aussi remarquables par leur capacité pulmonaire (ce qui peut résulter de leur vie en altitude doublée d'une intense activité nomade : on retrouve cette caractéristique chez certaines populations indiennes de la Cordillère des Andes et du Mexique). Des experts pensent que seuls les Kalenjins ont spécifiquement ces caractères physiques et que les coureurs Kisii qui sont leurs voisins doivent leurs qualités à la course aux mariages inter-tribaux de leurs ancêtres avec des femmes Nandi ou Kipsigis $! \gg{ }^{26}$.

Tous les avantages évoqués ici par Yves Pinaud, s'appuyant sur la morphologie, la physiologie ou la génétique, n’ont jamais été validés scientifiquement comme une explication de la domination de ces athlètes (Gaudin et Wolde, 2017 : XI). Les sciences de la vie tentent de mettre à l'épreuve ces discours, à travers des travaux en génétique (Scott and al. 2007 ; Wilber et Pitsiladis, 2012 : 92-102) ou des études comparatives entre des athlètes kényan.es et européen.nes en physiologie (Saltin, Larsen, Terrados et al., 1995 : 209-221 ; Prommer, Thoma, Quecke et al., 2010 : 791-797), sans toutefois aboutir à des résultats probants et des explications significatives. Malgré son absence de légitimité scientifique, ce type de discours médiatique a un retentissement important quant au regard porté par l'opinion publique sur les performances des athlètes est-africain·es. Comme c'est le cas en 1904 lors des Jeux anthropologiques de Saint-Louis, censés exacerber la suprématie de « la race blanche », « les médias alimentent l'imaginaire populaire » en attribuant un caractère racial aux performances physiques (Delsahut, 2010 : 685). A l'image des sportifs africains issus des colonies, les athlètes kényan·es ne doivent leurs succès qu'à des qualités naturelles aux yeux des journalistes, et non à des acquis culturels valorisés dans la société française (Jobert, 2006 : 192). Ainsi, si le discours racialiste autour de l'idéologie du don exprime la difficulté de se séparer d'une doctrine séculaire dans la période suivant les indépendances, sa pérennité jusqu'à la période actuelle témoigne de l'empreinte laissée par les discours orientalistes dans la culture des pays occidentaux $^{27}$ (Saïd, et al., 2005 : 19).

Bien que certains, comme Yves Pinaud ou Alain Billouin, s'attardent occasionnellement sur des facteurs socio-culturels pour expliquer les succès kényans, la « biologisation du social dans le sport » concernant les athlètes est-africain·es (Gaudin, 2017) est partagée par des revues et des auteurs aux profils variés. Vice-président du comité directeur de la FFA, André Gédéon écrit ainsi dans un article pour la revue fédérale que « ce sont les qualités naturelles qui permettent aux pays en voie de développement et sans passé athlétique d'atteindre le plus haut niveau $\gg^{28}$. Pour Charles Benejo, journaliste au Miroir de l'athlétisme : «Les Africains sont prédisposés à la réalisation 
de grandes performances athlétiques surtout dans les courses de vitesse, de demi-fond et de fond $\gg^{29}$. Dans cette dernière revue, le point de vue concernant l'idéologie du don est toutefois moins tranché que dans l'Équipe, Mahjoub Faouzi dénonçant ces clichés infondés qu'il attribue à des « négrologues ${ }^{30}$, sans citer directement son concurrent. De la même façon que le Miroir du cyclisme s'oppose à l'Équipe sur le sujet du dopage dans le cyclisme (Charroin, $2005: 6$ ), le Miroir de l'athlétisme semble vouloir se démarquer idéologiquement de son concurrent sur la perception des athlètes est-africain·es.

En lien avec cette vision du coureur « naturel », les athlètes kényans sont régulièrement dépeints par les journalistes comme instinctifs, naïf ${ }^{31}$ ou font l'objet de descriptions empruntes d'exotisme. Rédigés avec légèreté et ironie, on trouve, notamment dans la revue fédérale, habituellement moins portée sur l'athlétisme international, des commentaires sur la signification du nom de Kiprotich Rono (qui se traduirait par « le fils du vacher et de la bergère ») ou sur les dents manquantes d'Henri Rono $^{32}$. Des descriptions enfantines de coureurs kényans leur attribuent une certaine naïveté, comme à propos de Julius Korir ${ }^{33}$ que l'on surnomme « le petit kényan » ou « ce petit bonhomme de rien du tout [...] pouvant s'habiller au rayon garçonnet ». On parle également « des athlètes dits "naturels" » ${ }^{34}$, de « kényans de service ${ }^{35}$ ou de la « garde noire $»^{36}$. On peut aussi lire que Kipchogue Keino, de retour au Kenya après avoir étudié plusieurs années aux États-Unis « semble avoir retrouvé le naturel que la civilisation US avait provisoirement chassé ${ }^{37}$. Habituellement assignée aux animaux, la terminologie utilisée s'insère dans un « langage zoologique » usuel dans la période coloniale (Fanon, 2002 : 45), renvoyant à la vision rousseauiste du « mythe du bon sauvage » ou à celle de Georges Hébert concernant les « indigènes » (Bancel et Sirost, $2004:$ 396). Cette vision simpliste, caricaturale, selon laquelle les journalistes décrivent les coureurs kényans, caractéristique d'une position médiatique face à une population perçue comme naturellement dominée (Navarro et al., 2019: 6), reprend ici explicitement l'idéologie impérialiste civilisatrice. Utilisés par les journalistes français mais aussi par certains dirigeants fédéraux, les stéréotypes ${ }^{38}$ renvoient à « un imaginaire, de clichés et de fantasmes produits par l'héritage socioculturel de l'auteur » (Gaucher, 2008) à propos des sportifs africains, que l'on trouve aussi dans la littérature sportive de la première moitié du $\mathrm{XX}^{\text {ème }}$ siècle. La proximité avec la nature, les références exotiques, l'instinct animal ou le ton paternaliste sont autant de stéréotypes alors utilisés à l'égard des populations colonisées. Comme la presse sportive des années 1970, les métropolitain·es au début du $\mathrm{XX}^{\mathrm{ème}}$ siècle éprouvent de la curiosité envers les athlètes africains, s'intéressent à leurs modes de vie, tout en ayant des difficultés à dépasser les stéréotypes précédents (Deville-Danthu, 1997 : 204). Au-delà de leur ancrage culturel après les indépendances ${ }^{39}$, ces stéréotypes sont mobilisés par les journalistes français pour définir la valeur d'un athlétisme fondé sur des qualités naturelles moins valorisées que la culture technique de l'athlétisme français. Une telle stratégie leur permet de renforcer la communauté discursive entretenue avec leur lectorat (Bonnet, 2010) en s'extrayant d'une domination sportive qu'ils attribuent à des avantages biologiques jugés injustes (Bale, 2001 : 224-228). Al'instar du traitement médiatique réservé aux athlètes issus des colonies au début du $\mathrm{XX}^{\text {ème }}$ siècle, ce registre discursif est à l'origine du sentiment ambivalent des journalistes français à l'égard des athlètes kényans, puis kényanes, dans les années 1990.

\section{0-2000 : ENTRE REJET ET FASCINATION}

Dans les années 1990, « la supériorité absolue du Kenya $»^{40}$ en cross-country est admise par la presse française, qui parle du « rouleau compresseur kényan $»^{41}$, pour illustrer « l'ampleur de la domination du Kenya ${ }^{42}$, seulement talonné par l'Éthiopie. Les nombreuses confrontations entre les coureur·ses français·es et kényan·es révèlent un « fossé qui s'est creusé chez les séniors entre les crossmen kényans et européens ${ }^{43}$. Passionné d'histoire de l'athlétisme, membre des associations et commissions sur l'histoire de la FFA depuis les années $1960^{44}$, Alain Bouillé illustre ce qui apparaît comme une résignation avec nostalgie, en évoquant le titre mondial acquis en 1978 par l'équipe de France de cross-country. Il regrette ainsi que « depuis que les spécialistes africains (Kenya, Éthiopie) sont au départ (1981) du championnat du monde, un tel fait parait presque irréalisable ${ }^{45}$. Comme en cross-country, les courses sur route françaises, devenues l'apanage des athlètes kényan·es dans les années 1990, deviennent également représentatives du double sentiment qui anime les acteurs de l'athlétisme français à l'égard de ces athlètes. Pour Jacques Chanéac, rédacteur en chef de la revue Athlétisme : «on ne peut se réjouir de l'extrême discrétion des coureurs français dont on chercha continuellement la trace et des nouvelles durant les trois heures de retransmission en direct sur France $3 »^{46}$, après un marathon de Paris dominé par les coureurs africains. Un sentiment ambivalent de la part de la presse spécialisée française émerge vis-à-vis des athlètes africain.es participant aux courses sur route hexagonales, oscillant entre admiration et jalousie teintée de chauvinisme. Bien que se montrant parfois admiratifs à l'égard des athlètes est-africain·es, les journalistes se réjouissent de leur défaite face aux Français·es. Fidèles à la « tradition » du journalisme sportif, ils recourent à la métaphore pour magnifier les athlètes français·es tout en dépréciant leurs adversaires (Vanoudheusden, 2013). Dans un récit épique du marathon de Paris, Paul Miquel se satisfait ainsi qu': « Au $35^{\text {ème }}$ kilomètre, c'est Ouadi qui se prend pour D’Artagnan. Devant Roland Garros, poussé par une folle envie de vaincre, le "légionnaire volant" décroche du peloton de tête pour tenter l'aventure ! A partir de cet instant, les Kényans, Tanzaniens et autres 
sont lâchés, perdus, dégoûtés $»^{47}$. La performance du Français Abdellah Behar « neuvième du cross long au beau milieu d'une horde de kényans $»^{48}$ s'accompagne également de termes peu flatteurs pour ses rivaux. Cette ambivalence des médias rappelle certains faits sportifs du début du XX ${ }^{\text {ème }}$ siècle, telle la venue en France du cycliste noir américain Marshall Taylor, alors respecté et admiré par la presse sportive française. Les médias français couvrent pourtant son duel contre le Français Jacquelin en 1901 comme le duel entre « la race noire » et « la race blanche ». La victoire de Jacquelin sur celui que la presse française surnomme « le nègre volant », illustrant pour cette dernière la domination de la « race blanche » permettant de légitimer la colonisation ( Jobert, 2006 : 44-46). Si les propos des journalistes de la revue Athlétisme n'évoquent pas directement cette dimension 'raciale', le processus décrit précédemment dans lequel ils s'insèrent témoigne d'une volonté de s'extraire d'une domination qui dépasse la simple rivalité entre nations. Pour valoriser la onzième place de Paul Arpin derrière les athlètes kényans et éthiopiens, intouchables aux mondiaux de cross-country en 1988, Guy Manfredi, vice-président délégué au comité directeur de la FFA, lui attribue ainsi dans la revue fédérale une honorifique «première place des blancs ${ }^{49}$. Plus récemment, une telle opération est réalisée au lendemain du record de France du 10om de Christophe Lemaître, figeant le chronomètre sous les dix secondes, faisant titrer au journal l'Équipe $e^{50}:$ « Lemaître dans l'histoire », titre justifié par le fait que « le jeune français est devenu le premier athlète blanc à franchir à son tour cette barrière mythique $»^{51}$. Cette propension des journalistes à établir des classements alternatifs ségrégationnistes se traduit également à travers l'accueil favorable réservé à la création des championnats d'Europe de cross-country en 1994. Dans le contexte de domination des athlètes est-africain-es précédemment décrit, Francis Magois, journaliste pour Athlétisme avant de travailler pour l'Équipe, se satisfait que « les championnats d'Europe semblent arriver à point nommé pour redonner de la vitalité au cross du continent, passablement chahuté depuis l'arrivée au premier plan de l'invincible armada kenyane $\gg^{52}$. En continuité avec la période précédente, la bonne réception de cette compétition illustre la façon dont l'avantage naturel conféré aux athlètes africain -es par les Européen -nes légitime leur mise à distance (Denison, $2007: 53$ ). Ce type de réaction devant une domination jugée naturelle fait échos à la ferveur des journalistes hissant Georges Carpentier au rang de grand espoir de la boxe après son titre de « champion du monde de la race blanche » obtenu en 1914. ( Jobert, $2006: 83-86$ ).

\section{Conchusion}

Parallèlement aux succès des athlètes kényan·es, le discours journalistique évolue en conservant une relative homogénéité entre les revues analysées, et ce quelle que soit leur ligne éditoriale (et politique), et entre les auteurs, qu'ils soient journalistes ou acteurs fédéraux. L'ambivalence des auteurs ainsi que les croyances relatives à l'influence de l'environnement, en l'idéologie du don ainsi que les préjugés qui y sont associés sont des modèles discursifs déjà observés pendant la période coloniale, notamment au début du XXème siècle, concernant les sportifs africains. Les discours de presse analysés pérennisent donc après les indépendances la domination culturelle initiée dans une logique manichéenne par la littérature européenne pour justifier la colonisation ${ }^{53}$ (Ashcroft, Griffiths et Tiffin, 2003 : 124). Il semblerait ainsi y avoir de ce point de vue une filiation entre la période coloniale et la période post-coloniale, justifiant une analyse du point de vue des postcolonial studies. Ces discours ont néanmoins évolué depuis cette période, d'une part parce qu'ils intègrent (certes timidement) des variables socio-culturelles pour expliquer la domination des athlètes africain.es mais aussi parce qu'ils s'étendent à des populations non colonisées par la France. Enfin, si l'organisation du champ sportif dans la période coloniale est l'expression d'une lutte pour une domination socio-raciale, les discours journalistiques post-coloniaux étudiés, tout en mobilisant des stéréotypes racialistes, reflètent davantage la volonté de s'extraire d'une domination sportive jugée naturelle et injuste pour maintenir le lustre du demi-fond, considéré comme le « moteur de l'athlétisme français $»^{54}$.

Dépassant la sphère médiatique, ces stéréotypes se diffusent plus largement parmi les acteurs de l'athlétisme français, comme kényans, influençant leur organisation. Outre la mise à distance des athlètes estafricain·es dans les compétitions nationales ou internationales (Thomas et al., 2020), la démotivation de certains athlètes européen·nes, découragé·es par leurs défaites face aux athlètes kényan - es attribuées à des facteurs naturels incontrôlables (Baker et Horton 2003), ou la réappropriation par les athlètes kényan·es de stéréotypes les concernant, renforçant leur confiance en eux (Gaudin et Wolde 2017 : 235-236), en sont des exemples. Cette étude illustre finalement la vision antiréaliste du journalisme, dont l'information, basée sur l'itération d'une succession de réalités construites, ici selon un imaginaire postcolonial, génère de nouveaux faits sociaux (Gauthier, $2005: 54$ ).

Proposé le 15 juillet 2020 Accepté le 11 juin 2021 


\section{Notes}

1. Eliud Kipchoge, détenteur du record du monde officiel du marathon, réalise 1h59'40 dans une course d'exhibition organisée par la société Ineos où tout est optimisé pour courir un marathon en moins de deux heures, en s'affranchissant parfois des règles imposées par la fédération internationale (L'Équipe, 12 octobre 2019, pp. 4-5).

2. En dehors de l'Ougandais Joshua Cheptegei $(10 \mathrm{~km})$, toutes les autres performances sont réalisées par des athlètes kényan·es :

Geoffrey Kamworor (semi-marathon), Robert Keter (5km), Ronex Kipruto (10km), Brigid Kosgei (marathon féminin en course mixte) et Eliud Kipchoge.

3. Dans les bilans mondiaux, rassemblant les meilleures performances de tous les temps, sur les cents premier.es athlètes sur $10 \mathrm{~km}$, semi-marathon et marathon, les athlètes kényanes représentent respectivement $52 \%, 48 \%$ et $29 \%$ des athlètes ; les athlètes kényans représentent quant à eux $66 \%, 67 \%$ et $49 \%$ des athlètes. 4. Outre les avantages liés au projet d'Ineos et au marathon mixte, la presse sportive évoque régulièrement le dopage qui sévit parmi les athlètes kényan.es depuis quelques années et s'intéresse à de nouveaux modèles de chaussures qui conféreraient un avantage aux athlètes («A toutes pompes », Romain Donneux, L'Équipe, 18 octobre 2019, pp. 26-27).

5. «Le phénomène kényen », Y. Pinaud, L'Équipe athlétisme magazine ${ }^{\circ} 56$, Mai 1974, pp. 28-31.

6. Le marathonien passe devant l'obélisque d'Axoum, alors pillée à l'Éthiopie, avant de s'imposer sous l'arche Constantin d'où sont parties les troupes mussoliniennes qui ont colonisé l'Éthiopie en 1936, avant qu'elle ne retrouve son indépendance à l'issue de la seconde guerre mondiale. 
Disponible sur la plateforme de la BNF Gallica, l'intégralité des numéros de la revue Athlétisme parus entre 1960 et 2000 ont été consultés. La plupart des exemplaires des deux autres revues ont pu être consultés aux Archives nationales du monde du travail de Roubaix et grâce au concourt de collectionneurs auxquels j'exprime toute ma gratitude.

8. D'après plusieurs membres de la Commission de la Documentation et de l'Histoire de la Fédération Française d'Athlétisme présidée par Luc Vollard.

. Cette proximité s'illustre également à travers la mission FFA/l'Auto, menée en 1937 en Afrique Occidentale Française pour trouver des talents susceptibles de renforcer les rangs de l'athlétisme français, dans le cadre d'un partenariat entre le journal l'Auto (devenu l'Équipe) et la FFA (Frenkiel et Kemo Keimbou, 2010 : 33-50).

10. « Kenya terre de champions », Le Miroir de l'athlétisme n ${ }^{15}$, août 1965, pp.14-15.

11. « Et puis vint Keino », J. Velzian, Le Miroir de l'athlétisme n²9, mai 1967, pp. 7-9.

12. « Kenya : les champions du hasards », J. Viellvoye, Le Miroir de l'athlétisme n63, janvier 1970, pp. 8-13.

13. « Keino à cœur découvert », N. Couedel, L'Équipe athlétisme magazine $\mathrm{n}^{\circ} 6$, Mai 1969, pp. 32-34.

14. Le journaliste témoigne sa joie « de découvrir l'enfant que cachait le champion et de pénétrer tout à coup au beau milieu de ces histoires de tribus, de brousse africaine et de chasse ».

15. «L'explosion africaine », N. Couedel, L'Équipe athlétisme magazine $\mathrm{n}^{\circ} 32$, Septembre 1971, pp. 9-12.

16. Yves Pinaud travaille pour l'Équipe puis pour la revue l'Athlétisme. Statisticien spécialiste de l'athlétisme africain, il est notamment l'auteur de la série d'ouvrages annuels L'Athlétisme africain, compilant les résultats de l'athlétisme en Afrique depuis 1976.

17. «L'Afrique à huit mois des jeux », Y. Pinaud, L'Équipe athlétisme magazine $\mathrm{n}^{\circ} 34$, décembre 1971, pp. 23- 25.

18. « Lecteurs à vous de jouer », l'Équipe athlétisme magazine n³8 et 39, juin et juillet 1972 .

19. «Les Africains veulent prouver que Mexico n'était pas un accident », P. N’Doo, Le Miroir de l’Athlétisme n93, mai 1972, pp. 16-17.

20. « Comment résoudre la crise du demi-fond français », G. Meyer, L'Équipe athlétisme magazine n²12, octobre 1969, pp. 9-15. 21. «Afrique an 2 », M. Faouzi, Le Miroir de l'athlétisme n'101, mars 1973, pp. 5-7. Dans cet article, l'auteur ne cite pas directement le Kenya mais c'est alors le principal pays d'Afrique de l'Est ayant eu des résultats aux Jeux du Commonwealth, car anciennement sous giron britannique.

22. «Évolutions », J. Gilbert, L’Athlétisme n²17, mars 1977, p. 39.

23. Nous préférons ici le terme « racialiste » à « raciste » pour caractériser les articles évoquant l'idéologie du don car il s'agit davantage de stéréotypes reflétant l'idéologie d'une époque que de propos destinés à nuire (Todorov, $1989: 133-140$ ).

24. «Qu'est ce qui fait courir l'Afrique », A. Billouin, L'Équipe athlétisme magazine n50, Août 1973, pp. 32-34.

25. « Jeux du Commonwealth le défi africain », M. Hansenne, L'Équipe athlétisme magazine n52, Février 1974, pp. 15-17.

26. «Le phénomène kényen », op. cit.

27. Le succès de l'ouvrage du journaliste sportif américain Jon Entine, défendant plus récemment la thèse de l'existence d' « aptitudes athlétiques raciales naturelles » (Martin Breteau, $2010: 42$ ) illustre cette emprunte.

28. «Les petits, les obscurs, les sans grades... », A. Gédéon, Athlétisme n²63, septembre 1983, p. 65.

29. «Afrique : les dons ne sont pas tout », C. Benejo, Le Miroir de l'athlétisme n91, mars 1972, pp. 22-23.
30. « Afrique an $2 », o p$. cit.

31. « le phénomène kényen », op. cit.

32. Rubrique « échos », Athlétisme n²44, février 1982, p. 51.

33. Rubrique « Records », Athlétisme n²73, septembre 1984, p. 82.

34. «L'endurance en course à pied ( $3^{\mathrm{ème}}$ partie) », J. C. Colle, Athlétisme n²43, janvier 1982, p. 48.

35. «5000m Saïd Aouita avait choisi le $5000 »$, Athlétisme n²73, septembre 1984, p. 37.

36. « 1 ère coupe du monde de marathon Hiroshima 14 avril 1985 »,

G. Erckelbout, Athlétisme n²81, juin 1985, p. 23.

37. Rubrique « échos », Athlétisme n³08, février 1988, p. 41.

38. Le stéréotype désignant ici les croyances, opinions et représentations à propos d'un groupe et de ses membres selon trois caractéristiques : la résistance au changement, la propagation en dehors de toute réalité objective et la résultante d'un apprentissage social de savoirs partagés (Amossy et Herschberg Pierrot 2016 : 33-38).

39. Le chef d'État français Nicolas Sarkozy, lors d'une allocution à l'Université Cheikh Anta Diop de Dakar le 26 juillet 2007, fait référence à plusieurs reprises au lien entre $\mathrm{l}^{\prime}$ « Homme africain » et la nature qu'il oppose à «l'Homme moderne », affirmant notamment que «l'Homme moderne qui veut se réconcilier avec la nature a beaucoup à apprendre de l'Homme africain qui vit en symbiose avec la nature depuis des millénaires ».

40. « Championnats du monde de cross-country : l'Afrique aux africains », Y. Pinaud, Athlétisme n³89, avril 1996, p. 24.

41. «Le cross à l'heure européenne », F. Magois, Athlétisme n³71, mai 1994, p. 38.

42. « Championnats du monde de cross-country: A. Dias prive le Kenya du grand chelem », J. Chanéac, Athlétisme n³60, avril 1993, p. 33.

43. « Le cross à l'heure européenne », op. cit..

44. Alain Bouillé est membre de l'actuel Commission de la Documentation et de l'Histoire de la FFA.

45. « Les grands et grandes du cross-country », A. Bouillé, Athlétisme $\mathrm{n}^{\circ} 359$, mars 1993, p. 42.

46. « Marathon de Paris, championnats de France : Rémond, Tanigawa et 18000 autres », J. Chanéac, Athlétisme n³71, mai-juin 1994, p. 11.

47. « Mohamed Ouadi, 32000 derrières et lui devant », P. Miquel, Athlétisme n 429 , mai 2000, p. 11.

48. «Le joli printemps de Fatima Yvelain et d'Abdellah Behar », P. Miquel, Athlétisme n'429, mai 2000, p. 8.

49. "Championnats du monde de cross-country à Auckland (N-Z) : Annette Sergent et les français tous bronzés », G. Manfredi, Athlétisme n³11, mai 1988, p. 7 .

50. Une de L'Équipe du 10 juillet 2010.

${ }^{51 .}$ Le quotidien sportif qualifie cette performance d' « historique », alors qu'elle constitue à cette date la cinquante sixième performance de tous les temps et que Lemaitre n'est que le deuxième français à passer sous la barre des dix secondes (après Ronald Pognon).

52. « Le cross à l'heure européenne », op. cit.

53. Voir Kipling, R., 1989. « Le Fardeau de l'homme

Blanc », McClure's Magazine $\mathrm{n}^{\circ} 12$.

54. « Comment résoudre la crise du demi-fond français », op. cit. 


\section{BIBLIOGRAPHIE}

Amossy, R. \& Herschberg Pierrot, A. (2016). Stéréotypes et clichés, langue discours, société. Paris : Armand Colin.

Ashcroft, B., Griffiths, G. \& Tiffin, H. (2003). The Empire Writes Back: Theory and Practice in Post-Colonial Literatures. London: Routledge.

Ashcroft, B., Griffiths, G. \& Tiffin, H. (2013). Post-colonial studies: The key concepts. Oxford: Routledge.

Attali, M. (2018). La presse sportive : militantisme, socialisation et modes d'appréhension du réel. In Blandin, C. (Eds.), Manuel d'analyse de la presse magazine (pp. 179-190). Paris : Armand Colin.

Baker, J. \& Horton, S. (2003). "East African running dominance revisited: a role for stereotype threat?". British Journal of Sports Medicine, vol.37, n6, pp. 553-555.

Bale, J. \& Sang, J. (1996). Kenyan Running: Movement Culture, Geography and Global Change, London: Routledge

Bale, J. (2001). Nyandika Maiyoro and Kipchoge Keino: transgression, colonial rhetoric and the postcolonial athlete. In Andrews D. L. \& Jackson S. J. (Eds.), Sport Stars: The Cultural Politics of Sporting Celebrity (pp. 218-230). London: Routledge.

Bale, J. (2002). "Lassitude and latitude: Observations on sport and environmental determinism", International Review for the Sociology of Sport, vol.37, n², pp. 147-158.

Bale, J. (2007). Kenyan running before the 1968 Mexico Olympics. In Pitsiladis, Y., Bale, J., Sharp, C. \& Noakes, T. (Eds.), East African Running Toward a Cross Disciplinary Perspective (pp. 35-47). New-York: Routledge.

Bancel, N. \& Blanchard P. (2006). Culture post-coloniale : le temps des héritages. In Bancel, N., Blanchard, N. (Eds.), Culture post-coloniale : traces et mémoires coloniales en France (pp. 6-19). Paris : Autrement.

Bancel, N. \& Sirost, O. (2004). Le corps de l'autre : une nouvelle économie du regard. In Bancel, N. (Eds.), Zoos humains. Au temps des exhibitions humaines (pp. 390-398). Paris : La Découverte.

Bazoge, N., \& Jamain-Samson, S. (2013). Les championnes de Miroir Sprint (1967-1970) : des modèles de « filles réussies ». In Attali, J. \& Combeau-Mari, E. (Eds.), Le sport dans la presse communiste (pp. 231-248). Rennes : Presse Universitaire de Rennes.

Bonnet, V., (2010). « Les stéréotypes dans la presse sportive : vision de l'identité à travers l'altérité », Signes, discours et société, vol. $11, \mathrm{n}^{\circ} 4$.

Charroin, P., (2005). « L'affaire Simpson de 1967 : une rupture médiatique dans l'appréhension du dopage », Éthique publique. Revue internationale d'éthique sociétale et gouvernementale, vol.7, n², pp. 1-14.

Delsahut, F. (2010). Sport, race et médias aux Jeux anthropologiques de Saint-Louis en 1904. In Attali, M. (Eds.), Sports et médias : du XIXe siècle à nos jours (pp. 677-690). Biarritz : Atlantica.

Denison, J. (2007). The Haile Gebrselassie story: A biography of difference. In Pitsiladis, Y., Bale, J., Sharp, C. \& Noakes, T. (Eds.), East African Running Toward a Cross Disciplinary Perspective (pp. 51-59). New-York: Routledge.
Deville-Danthu, B. (1997). Le Sport en Noir et Blanc : du Sport Colonial au Sport Africain dans les Anciens Territoires Français d'Afrique Occidentale (1920-1965). Paris : L'Harmattan.

Duret, P. (1993). L’hérö̈sme sportif. Paris : Presse universitaire de France.

Fanon, F. (2002). Les damnés de la Terre. Paris : La Découverte.

Farge, A. (1989). Le goût de l'archive. Paris : Le Seuil.

Forté, L. (2019). « Stéréotypes "raciaux" dans la presse sportive : Une analyse des mécanismes conduisant à la production d'énoncés "racialisants" », Questions de communication, vol. $35, n^{\circ} 1$, pp. 63-81.

Frenkiel, S. \& Kemo Keimbou, D. C. (2010). « La Mission FFA/L'Auto : "Pourquoi négliger nos noirs d'Afrique ? "” (3 décembre 1937-15 janvier 1938) », Modern \& Contemporary France, vol.18, ${ }^{\circ} 1$, pp. 33-50.

Gaucher, J. (2008). Sport et genre : quand la littérature s'en mêle : féminités et masculinités dans l'écriture littéraire du sport (1920-1955). Thèse de doctorat en littérature française, sous la direction de Terret, T. \& Vray, J. B., Université Claude Bernard Lyon 1, 848 p.

Gaudin, B. (2008). « Athlétisme et nationalisme dans l'Éthiopie des années 1920-1960 », Sciences sociales et sport, $\mathrm{n}^{\circ} 1$, pp. 49-78.

Gaudin, B. (2017). Biologisation du social dans le sport : l' « athlète naturel est-africain ». In Lemerle, S. \& Paligot, C. (Eds.), La biologisation du social. Discours et pratiques, Paris : Presse universitaire de Paris Nanterre.

Gaudin, B. \& WOLDE, B. (2017). Kenyan and Ethiopian athletics: towards an alternative scientific approach. Addis Ababa: IRD/CFEE.

Gauthier, G. (2005). "A realist point of view on news journalism”, Journalism Studies, vol.6, n¹, pp. 51-60.

Jobert, T. (2006). Champions Noirs, Racisme Blanc : La Métropole et les Sportifs Noirs en Contexte Colonial (1901-1944). Grenoble : Presse Universitaire de Grenoble.

Kasperowski, D. (2009). "Constructing altitude training standards for the 1968 Mexico Olympics: the impact of ideals of equality and uncertainty", The International Journal of the History of Sport, vol.26, ${ }^{\circ}$ 9, pp. 1263-1291.

Krieg, A. (2000), « Analyser le discours de presse. Mises au point sur le "discours de presse" comme objet de recherche ", Communication. Information médias théories pratiques, vol.20, $\mathrm{n}^{\circ} 1$, pp. 75-97.

Lacoste, Y. (2006). « La question postcoloniale », Hérodote, vol. $120, \mathrm{n}^{\circ} 1$, pp. 5-27.

L'Heuillet, H. (2017). « Les études postcoloniales, une nouvelle théorie de la domination ? », Cités, vol. 4, n 4, pp. 4152.

Manners, J. (1997). “Kenya's running tribe”, The Sports Historian, vol.17, n², pp. 14-27.

Martin-Breteau, N. (2010). «"Un laboratoire parfait” ? Sport, race et génétique : le discours sur la différence athlétique aux États-Unis », Sciences sociales et sport, 1, pp. 7-43. 
Martin-Breteau, N. (2013). « Sport, race, et politique : Taboo et la réception du discours sur les aptitudes athlétiques des races aux États-Unis », Le mouvement social, 1, pp. 131147.

Navarro, L., Ross, K. \& Saitta, E. (2019). « Stéréotypes dans l'exercice du journalisme », Sur le journalisme, About journalism, Sobre jornalismo, vol.8, n², pp. 6-11.

Pociello. C. (1998). « Sur la dramaturgie des jeux de combat », Communications, vol. 67, n¹, pp. 149-164.

Prommer, N., Thomas, S., Quecke, L. \& al. (2010). “Total hemoglobin mass and blood volume of elite Kenyan runners”, Medicine and science in sports and exercise, vol.42, $\mathrm{n}^{\circ} 4$, pp. 791-797.

Provenzano, F. (2018). Le discours de la presse magazine. In Blandin, C. (Eds.), Manuel d'analyse de la presse magazine (pp. 85-104). Paris : Armand Colin.

Sabatier, F. (2013). La presse sportive affinitaire et les trois grands engagements coloniaux des organisations communistes de France. In Attali, J. \& Combeau-Mari, E. (Eds.), Le sport dans la presse communiste (pp. 91-100). Rennes : Presse Universitaire de Rennes.

Said, E. W., Malamoud, C., Meininger, S., \& al. (2005). L'Orientalisme : l'Orient Créé par l'Occident. Paris : Seuil.

Saltin, B., Larsen, H., Terrados, N. \& al. (1995). "Aerobic exercise capacity at sea level and at altitude in Kenyan boys, junior and senior runners compared with Scandinavian runners", Scandinavian Journal of Medicine \& Science in Sports, vol.5, n4, pp. 209-221.

Savarese, E. (2014). La rencontre postcoloniale. Bellecombeen-Bauges : éditions du Croquant.

Scott, R. A., Goodwin, W. H., Wolde, B., Onywera, V. O., Boit, M., O’Connell, W. \& Pitsalidis, Y. (2007). Evidence for the "natural" east African athlete. In Pitsiladis, Y., Bale, J., Sharp, C. \& Noakes, T. (Eds.), East African Running Toward a Cross Disciplinary Perspective (pp. 257-282). New-York: Routledge.

Seidler, E. (1964). Le sport et la presse. Paris : Armand Colin. Smouts, M. (2007). La situation postcoloniale : Les postcolonial studies dans le débat français. Paris : Presses de Sciences Po.

Souanef, K. (2019). Le journalisme sportif. Sociologie d'une spécialité dominée. Rennes : Presses universitaires de Rennes.

Thomas, C., Charroin, P. \& Soulé, B. (2021). « De la curiosité à l'évitement : l'adaptation des instances françaises de l'athlétisme face à la domination du Kenya en course de fond », Sciences Sociales et Sport, n 17, pp. 87-112.

Thomas, C., Charroin, P. \& Soulé, B. (2020). « Les relations franco-kényanes dans les courses de fond: un processus postcolonial singulier (1960-2019) », Stadion. International Journal of the History of Sport, vol. 44, n 1, pp. 204-225.

Todorov, T. (1989). Nous et les autres. La réflexion française sur la diversité humaine. Paris: Seuil.

Vanoudheusden, R. (2013). Comme les journalistes sportifs nous parlent : étude d'un lexique « hyper-appréciatif ». In Abecassis, M. \& Ledegen, G. (Eds.), Écarts et apports des médias francophones (pp. 227-244), Oxford : Peter Lang.

Wiggins, D. K. (1989). " "Great Speed But Little Stamina”: The Historical Debate Over Black Athletic Superiority », Journal of Sport History, vol.16, n², pp. 158-185.

Wilber, R. L. \& Pitsiladis, Y. (2012). "Kenyan and Ethiopian distance runners: what makes them so good", International Journal of Sports Physiology and Performance, vol.7, n², pp. 92-102. 


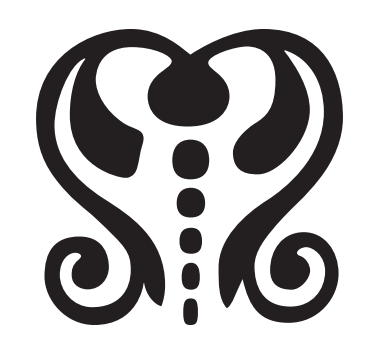




\section{RÉSUmé | RESUmo | AbSTRaCT}

Un processus postcolonial : la genèse de la domination kényane dans les courses de
fond vue par la presse sportive spécialisée française

Um processo pós-colonial: a gênese da dominação queniana em corridas de longa distância vista pela imprensa esportiva especializada francesa

A postcolonial process: The genesis of Kenyan preeminence in long-distance running, as seen by the French sports press.

Fr. Les médias se font régulièrement l'échos des exploits, sinon des dérives, des athlètes kényan·e.s qui dominent les épreuves de course de fond les plus prestigieuses à tra- vers le monde. Désormais coutumière de cette hégémonie, la presse sportive française commence à l'interroger dès les années 1960, tandis que l'athlétisme est-africain s'affirme au plus haut niveau international, manifestant sa volonté de comprendre et d'expliquer le «phénomène kényan ». L'objet de cet article est de montrer que l'éclosion au plus haut niveau international de l'athlétisme kényan dans la période post-coloniale est appréhendée par les journalistes français selon une rhétorique s'insérant dans un processus postcolonial. S'inscrivant dans le champ des postcolonial studies, cette étude vise à identifier et expliquer les transformations des modalités discursives selon lesquelles les journalistes français couvrent les succès kényans. Bien que le Kenya soit une ancienne colonie britannique, les textes étudiés reflétent la domination culturelle caractéristique de la période coloniale que les journalistes opposent à la domination sportive des athlètes kényan·e·s. Trois revues spécialisées dans l'athlétisme paraissant dans les années 1960, choisies tant par leur réputation que par l'éclectisme de leurs lignes éditoriales, sont analysées : l'Athlétisme, organe de presse officiel de la Fédération française d'athlétisme, Le Miroir de l'athlétisme, revue déclinée du journal Miroir sprint, proche du parti communiste français, et l'Équipe athlétisme magazine, associée au journal l'Équipe. Portant sur l'ensemble du discours, tant son contenu que ses stratégies énonciatives, l'analyse effectuée met en avant le recours par les journalistes français aux modèles rhétoriques utilisés par leurs homologues britanniques à la fin de la période coloniale. Identifiés par John Bale, ces modèles rhétoriques (la surveillance, l'appropriation, la négation et l'idéalisation) s'affirment progressivement à travers quatre étapes chronologiques de 1960 à 2000. La France n'ayant jamais colonisé le Kenya, cette démarche propose donc d'élargir la question postcoloniale aux interactions culturelles entre des pays dépourvus de liens coloniaux.

Mots clés : Postcolonial - Athlétisme - Kenya - journalisme - domination

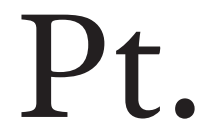

Os meios de comunicação informam regularmente sobre as façanhas, senão os desvios, dos atletas quenianos que dominam os eventos de corrida de longa distância de maior prestígio em todo o mundo. Já habituada a esta hegemonia, a imprensa desportiva francesa passou a questioná-la nos anos 1960, enquanto o atletismo da África Oriental se afirmava ao mais alto nível internacional, demonstrando o seu desejo de compreender e explicar o "fenômeno queniano". O objetivo deste artigo é mostrar que a emergência do atletismo queniano no mais alto nível internacional no período pós-colonial é entendida pelos jornalistas franceses como uma parte retórica de um processo pós-colonial. Inserido no campo dos estudos pós-coloniais, este estudo visa identificar e explicar as transformações das modalidades discursivas segundo as quais os jornalistas franceses cobrem os sucessos quenianos. Embora o Quênia seja uma ex-colônia britânica, os textos estudados refletem a dominação cultural característica do período colonial que os jornalistas opõem à dominação esportiva dos atletas quenianos. Três revistas especializadas em atletismo surgidas na década de 1960, escolhidas tanto por sua reputação quanto pelo ecletismo de suas linhas editoriais, são analisadas: Athletics, órgão oficial de imprensa da Federação Francesa de Atletismo, Le Miroir de athletics, resenha da revista Miroir sprint, próxima ao Partido Comunista Francês, e a revista de atletismo Équipe, associada ao jornal L'Équipe. Abrangendo todo o discurso, tanto o seu conteúdo como as suas estratégias enunciativas, a análise realizada destaca a 
utilização pelos jornalistas franceses dos modelos retóricos utilizados pelos seus congêneres britânicos no final do período colonial. Identificados por John Bale, esses modelos retóricos (vigilância, apropriação, negação e idealização) afirmam-se gradativamente por meio de quatro estágios cronológicos de 1960 a 2000. Como a França nunca colonizou o Quênia, esta abordagem propõe, portanto, estender a questão pós-colonial às interações culturais entre países desprovidos de laços coloniais.

Palavras-Chave: Pós-colonial; atletismo; Quênia; jornalismo; dominação

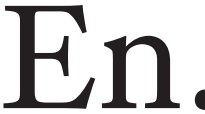

Media regularly report on the high performances, as well as the missteps, of Kenyan athletes occupying top places in the most prestigious international long-disto this podium hegemony, a desire to understand and explain the "Kenyan phenomenon" arose in the 1960's, when East African athletics was gradually asserting itself at the highest levels of competition. The article aims to demonstrate that the narrative developed by French journalists on Kenyan athletics in the decades following African independences is part of rhetorical processes intertwined with postcolonial mechanisms. Embedded in the field of postcolonial studies, the research aims to identify and explain the evolution of discursive modalities used by French journalists to cover Kenyan sporting successes. Despite Kenya being a former British colony, specific characteristics appear in the analyzed text corpus and highlight how French journalists perpetuate cultural domination mechanisms, which sit in contrast with the sporting preeminence of Kenyan athletes. Three magazines published in the 1960 os and specialized in the field of athletics were selected for the research, based on their reputation and the eclecticism of their editorial lines: L'Athlétisme, published by the French Athletics Federation; Le Miroir de l'athlétisme, a magazine based on the Miroir sprint, known to be close to the French Communist Party ; and l'Équipe athlétisme magazine, a spin-off edition from the newspaper l'Équipe. The analysis of the discourse, from both content and enunciative strategies perspectives, highlights how French journalists resort to rhetorical models used by their British counterparts during the same period. Identified by John Bale, the models include surveillance, appropriation, negation and idealization, and appear chronologically in the press in four stages, from 1960 to 2000. Since Kenya was never under French colonial rule, the article suggests to broaden the postcolonial discussion to cultural interactions between countries without colonial ties.

Key words: Postcolonial - Athletics - Kenya - journalism - domination 